\title{
Certain combinatoric Bernoulli polynomials and convolution sums of divisor functions
}

Daeyeoul Kim ${ }^{1}$ and Nazli Yildiz Ikikardes²

${ }^{\text {"Correspondence: }}$

nyildizikikardes@gmail.com

2Department of Elementary Mathematics Education, Necatibey

Faculty of Education, Balikesir University, Balikesir, 10100, Turkey

Full list of author information is

available at the end of the article

\begin{abstract}
It is known that certain convolution sums can be expressed as a combination of divisor functions and Bernoulli formula. One of the main goals in this paper is to establish combinatoric convolution sums for the divisor sums $\hat{\sigma}_{s}(n)=\sum_{d \mid n}(-1)^{\frac{n}{d}-1} d^{s}$. Finally, we find a formula of certain combinatoric convolution sums and Bernoulli polynomials.
\end{abstract}

MSC: 11A05; 33E99

Keywords: Bernoulli numbers; convolution sums

\section{Introduction}

The symbols $\mathbb{N}$ and $\mathbb{Z}$ denote the set of natural numbers and the ring of integers, respectively. The Bernoulli polynomials $B_{k}(x)$, which are usually defined by the exponential generating function

$$
\frac{t e^{x t}}{e^{t}-1}=\sum_{k=0}^{\infty} B_{k}(x) \frac{t^{k}}{k !},
$$

play an important and quite mysterious role in mathematics and various fields like analysis, number theory and differential topology. The Bernoulli polynomials satisfy the following well-known identities:

$$
\begin{aligned}
\sum_{j=0}^{N} j^{k} & =\frac{B_{k+1}(N+1)-B_{k+1}(0)}{k+1} \quad(k \geq 1) \\
& =\frac{1}{k+1} \sum_{j=0}^{k}(-1)^{j}\left(\begin{array}{c}
k+1 \\
j
\end{array}\right) B_{j} N^{k+1-j} .
\end{aligned}
$$

The Bernoulli numbers $B_{k}$ are defined to be $B_{k}:=B_{k}(0)$. For $n \in \mathbb{N}, k \in \mathbb{Z}$, we define some divisor functions

$$
\begin{aligned}
& \sigma_{k}(n):=\sum_{d \mid n} d^{k}, \quad \sigma_{k}^{*}(n):=\sum_{\substack{d \mid n \\
\frac{n}{d} \text { odd }}} d^{k}, \quad \widetilde{\sigma}_{k}(n):=\sum_{d \mid n}(-1)^{d-1} d^{k}, \\
& \hat{\sigma}_{k}(n):=\sum_{d \mid n}(-1)^{\frac{n}{d}-1} d^{k}, \quad \sigma_{k, l}(n ; 2):=\sum_{\substack{d \mid n \\
d \equiv l \bmod 2)}} d^{k} .
\end{aligned}
$$

O2013 Kim and Yildiz Ikikardes; licensee Springer. This is an Open Access article distributed under the terms of the Creative Commons Attribution License (http://creativecommons.org/licenses/by/2.0), which permits unrestricted use, distribution, and reproduction in any medium, provided the original work is properly cited. 
It is well known that $\sigma_{k}^{*}(n)=\sigma_{k}(n)-\sigma_{k}\left(\frac{n}{2}\right)$ and $\hat{\sigma}_{k}(n)=\sigma_{k}(n)-2 \sigma_{k}\left(\frac{n}{2}\right)[1,(1.13)]$. The identity

$$
\sum_{k=1}^{n-1} \sigma(k) \sigma(n-k)=\frac{5}{12} \sigma_{3}(n)+\left(\frac{1}{12}-\frac{1}{2} n\right) \sigma(n)
$$

for the basic convolution sum first appeared in a letter from Besge to Liouville in 1862 [2]. Hahn $[1,(4.8)]$ considered

$$
36 \sum_{m<n} \hat{\sigma}(m) \hat{\sigma}(n-m)= \begin{cases}-3 \hat{\sigma}(n)+3 \widetilde{\sigma}_{3}(n) & \text { if } n \text { is odd } \\ -3 \hat{\sigma}(n)-5 \widetilde{\sigma}_{3}(n)+4 \widetilde{\sigma}_{3}\left(\frac{n}{2}\right) & \text { if } n \text { is even. }\end{cases}
$$

For some of the history of the subject, and for a selection of these articles, we mention $[3,4]$ and [5], and especially [6, 7] and [8]. The study of convolution sums and their applications is classical, and they play an important role in number theory. In this paper, we investigate the combinatorial Bernoulli numbers and convolution sums. For $k$ and $n$ being positive integers, we show that the sum

$$
\sum_{j=2}^{k}\left(\begin{array}{c}
2 k+1 \\
2 j
\end{array}\right) B_{2 j} \hat{\sigma}_{2 k+1-2 j}(n)
$$

can be evaluated explicitly in terms of divisor functions and a combinatorial convolution sum. We prove the following.

Theorem 1 Let $k, n$ be positive integers. Then

$$
\begin{aligned}
\sum_{j=2}^{k}\left(\begin{array}{c}
2 k+1 \\
2 j
\end{array}\right) B_{2 j} \hat{\sigma}_{2 k+1-2 j}(n) \\
=(2 k+1) \sigma_{2 k+1}^{*}(n)-\left(\frac{2 k+3}{2}\right) \hat{\sigma}_{2 k+1}(n)-\left(\frac{k(2 k+1)}{6}\right) \hat{\sigma}_{2 k-1}(n) \\
\quad-(2 k+1) \sum_{s=0}^{k-1}\left(\begin{array}{c}
2 k \\
2 s+1
\end{array}\right) \sum_{m=1}^{n-1} \hat{\sigma}_{2 k-2 s-1}(m) \hat{\sigma}_{2 s+1}(n-m) .
\end{aligned}
$$

Remark 2 Let $n$ be positive integers. In Theorem 1, replace $k$ by 1 , we find easily that

$$
\sum_{m=1}^{n-1} \hat{\sigma}(m) \hat{\sigma}(n-m)=\frac{1}{2} \sigma_{3}^{*}(n)-\frac{5}{12} \hat{\sigma}_{3}(n)-\frac{1}{12} \hat{\sigma}(n)
$$

and in particular, if $q \in N, p=2 q+1$, an odd prime integer, then

$$
\sum_{m=1}^{q} \hat{\sigma}(m) \hat{\sigma}(p-m)=\frac{1}{6} q(q+1)(2 q+1)=\sum_{m=1}^{q} k^{2}=\frac{1}{3} B_{3}(q+1) .
$$

Equations (1.3) and (1.4) are in (1.2) and [9, Corollary 2.4]. Using these combinatoric convolution sums, we obtain the following. 
Theorem 3 If $k$ is a positive integer, then

$$
\sum_{u+v+w=2 k+1} 2^{v-2 k-1}\left(\begin{array}{c}
2 k+1 \\
u, v, w
\end{array}\right) B_{v} \cdot(2 l+1)^{w}=B_{2 k+1}(l+1),
$$

where $\left(\begin{array}{c}2 k+1 \\ u, v, w\end{array}\right)=\frac{(2 k+1) !}{u ! v ! w !}$ and $l=1,2,3,4,5,6,7,8,9,10,11,12$.

Thus, we can pose a general question regarding Bernoulli polynomials.

Question For all $k, l \in \mathbb{N}$, does the identity

$$
\sum_{u+v+w=2 k+1} 2^{v-2 k-1}\left(\begin{array}{c}
2 k+1 \\
u, v, w
\end{array}\right) B_{v} \cdot(2 l+1)^{w}=B_{2 k+1}(l+1) \quad \text { hold? }
$$

The problem of convolution sums of the divisor function $\sigma_{1}(n)$ and the theory of Eisenstein series has recently attracted considerable interest with the emergence of quasimodular tools. In connection with the classical Jacobi theta and Euler functions, other aspects of the function $\sigma_{1}(n)$ are explored by Simsek in [10]. Finally, we prove the following.

Theorem 4 If $a(\geq 2)$ and $k$ are positive integers, then

(i)

$$
\begin{aligned}
& \sum_{s=0}^{k-1}\left(\begin{array}{c}
2 k \\
2 s+1
\end{array}\right) \sum_{m=1}^{2^{a}-1} \sigma_{2 k-2 s-1,1}\left(\frac{m}{2} ; 2\right) \sigma_{2 s+1}\left(2^{a}-m\right) \\
& \quad=\left(\frac{1}{2(2 k+1)}\right) B_{2 k+1}\left(2^{a}\right)+\left(\frac{1-2^{2 k}}{2(2 k+1)}\right) \sum_{i=1}^{a-1} B_{2 k+1}\left(2^{i}\right)+\frac{1}{4}\left(\frac{2^{(2 k+1) a}-1}{2^{2 k+1}-1}-2^{a+1}+1\right),
\end{aligned}
$$

(ii)

$$
\begin{aligned}
\sum_{s=0}^{k-1}\left(\begin{array}{c}
2 k \\
2 s+1
\end{array}\right) \sum_{m=1}^{2^{a}-1} \sigma_{2 k-2 s-1,0}\left(\frac{m}{2} ; 2\right) \sigma_{2 s+1}\left(2^{a}-m\right) \\
=\left(\frac{1+2^{2 k}}{2(2 k+1)}\right) \sum_{i=1}^{a-1} B_{2 k+1}\left(2^{i}\right)+\frac{1}{4}\left(\frac{2^{(2 k+1) a}-1}{2^{2 k+1}-1}\right) \\
+\frac{1}{2}\left(\frac{2^{2 k(a+1)}-2^{2 k(a+1)-1}+2^{2 k a-1}-1}{2^{2 k}-1}\right) \\
+2^{a-2}\left(\frac{3-2^{(2 k-1)(a+1)}-2^{(2 k-1) a+1}}{2^{2 k-1}-1}\right)+2^{a-1}-\frac{1}{4},
\end{aligned}
$$

(iii)

$$
\begin{aligned}
& \sum_{s=0}^{k-1}\left(\begin{array}{c}
2 k \\
2 s+1
\end{array}\right) \sum_{m=1}^{2^{a}-1} \sigma_{2 k-2 s-1}\left(\frac{m}{2}\right) \sigma_{2 s+1}\left(2^{a}-m\right) \\
& \quad=\left(\frac{1}{2(2 k+1)}\right) B_{2 k+1}\left(2^{a}\right)+\left(\frac{1}{(2 k+1)}\right) \sum_{i=1}^{a-1} B_{2 k+1}\left(2^{i}\right)
\end{aligned}
$$




$$
\begin{aligned}
& +\frac{1}{2}\left(\frac{2^{(2 k+1) a}-1}{2^{2 k+1}-1}\right)+\frac{1}{2}\left(\frac{2^{2 k(a+1)}-2^{2 k(a+1)-1}+2^{2 k a-1}-1}{2^{2 k}-1}\right) \\
& +2^{a-2}\left(\frac{3-2^{(2 k-1)(a+1)}-2^{(2 k-1) a+1}}{2^{2 k-1}-1}\right) .
\end{aligned}
$$

\section{Properties of convolution sums derived from divisor functions}

Proposition 5 ([8]) Let $k, n$ be positive integers. Then

$$
\begin{aligned}
\sum_{j=2}^{k}\left(\begin{array}{c}
2 k+1 \\
2 j
\end{array}\right) B_{2 j} \sigma_{2 k+1-2 j}(n)= & -\left(\frac{2 k+3}{2}\right) \sigma_{2 k+1}(n)-(2 k+1)\left(\frac{k}{6}-n\right) \sigma_{2 k-1}(n) \\
& +(2 k+1) \sum_{s=0}^{k-1}\left(\begin{array}{c}
2 k \\
2 s+1
\end{array}\right) \sum_{m=1}^{n-1} \sigma_{2 k-2 s-1}(m) \sigma_{2 s+1}(n-m) .
\end{aligned}
$$

Proposition $6([7,11])$ Let $k, n$ be positive integers. Then

$$
\begin{aligned}
& \text { (i) } \sum_{s=0}^{k-1}\left(\begin{array}{c}
2 k \\
2 s+1
\end{array}\right) \sum_{m=1}^{n-1} \sigma_{2 k-2 s-1}^{*}(m) \sigma_{2 s+1}^{*}(n-m)=\frac{1}{2}\left(\sigma_{2 k+1}^{*}(n)-n \sigma_{2 k-1}^{*}(n)\right), \\
& \text { (ii) } \sum_{s=0}^{k-1}\left(\begin{array}{c}
2 k \\
2 s+1
\end{array}\right) \sum_{m=1}^{n} \sigma_{2 k-2 s-1}(2 m-1) \sigma_{2 s+1}(2 n-2 m+1)=\frac{1}{4} \sigma_{2 k+1}^{*}(2 n) .
\end{aligned}
$$

Proof of Theorem 1 Let $k, n \in \mathbb{N}$. By Proposition 5 and Proposition 6, we obtain

$$
\begin{aligned}
T:= & \sum_{s=0}^{k-1}\left(\begin{array}{c}
2 k \\
2 s+1
\end{array}\right) \sum_{m=1}^{n-1} \hat{\sigma}_{2 k-2 s-1}(m) \hat{\sigma}_{2 s+1}(n-m) \\
= & \sum_{s=0}^{k-1}\left(\begin{array}{c}
2 k \\
2 s+1
\end{array}\right) \sum_{m=1}^{n-1}\left(\sigma_{2 k-2 s-1}(m)-2 \sigma_{2 k-2 s-1}\left(\frac{m}{2}\right)\right) \\
& \times\left(\sigma_{2 s+1}(n-m)-2 \sigma_{2 s+1}\left(\frac{n-m}{2}\right)\right) .
\end{aligned}
$$

It is easily checked that

$$
\begin{aligned}
& \sigma_{2 k-2 s-1}\left(\frac{m}{2}\right) \sigma_{2 s+1}(n-m)+\sigma_{2 k-2 s-1}(m) \sigma_{2 s+1}\left(\frac{n-m}{2}\right) \\
& =\sigma_{2 k-2 s-1}\left(\frac{m}{2}\right) \sigma_{2 s+1}\left(\frac{n-m}{2}\right)+\sigma_{2 k-2 s-1}(m) \sigma_{2 s+1}(n-m) \\
& \quad+\left(\sigma_{2 k-2 s-1}(m)-\sigma_{2 k-2 s-1}\left(\frac{m}{2}\right)\right)\left(\sigma_{2 s+1}(n-m)-\sigma_{2 s+1}\left(\frac{n-m}{2}\right)\right) .
\end{aligned}
$$

Thus,

$$
\begin{aligned}
T= & \sum_{s=0}^{k-1}\left(\begin{array}{c}
2 k \\
2 s+1
\end{array}\right) \sum_{m=1}^{n-1}\left(\sigma_{2 k-2 s-1}(m) \sigma_{2 s+1}(n-m)+4 \sigma_{2 k-2 s-1}\left(\frac{m}{2}\right) \sigma_{2 s+1}\left(\frac{n-m}{2}\right)\right) \\
& -2 \sum_{s=0}^{k-1}\left(\begin{array}{c}
2 k \\
2 s+1
\end{array}\right) \sum_{m=1}^{n-1}\left(\sigma_{2 k-2 s-1}\left(\frac{m}{2}\right) \sigma_{2 s+1}\left(\frac{n-m}{2}\right)+\sigma_{2 k-2 s+1}(m) \sigma_{2 s+1}(n-m)\right)
\end{aligned}
$$




$$
\begin{aligned}
& -2 \sum_{s=0}^{k-1}\left(\begin{array}{c}
2 k \\
2 s+1
\end{array}\right) \sum_{m=1}^{n-1}\left(\sigma_{2 k-2 s-1}(m)-\sigma_{2 k-2 s-1}\left(\frac{m}{2}\right)\right) \\
& \times\left(\sigma_{2 s+1}(n-m)-\sigma_{2 s+1}\left(\frac{n-m}{2}\right)\right) \\
& =2 \sum_{s=0}^{k-1}\left(\begin{array}{c}
2 k \\
2 s+1
\end{array}\right) \sum_{m=1}^{n-1}\left(\sigma_{2 k-2 s-1}(m)-\sigma_{2 k-2 s-1}\left(\frac{m}{2}\right)\right)\left(\sigma_{2 s+1}(n-m)-\sigma_{2 s+1}\left(\frac{n-m}{2}\right)\right) \\
& -\sum_{s=0}^{k-1}\left(\begin{array}{c}
2 k \\
2 s+1
\end{array}\right) \sum_{m=1}^{n-1}\left(\sigma_{2 k-2 s-1}(m) \sigma_{2 s+1}(n-m)-2 \sigma_{2 k-2 s-1}\left(\frac{m}{2}\right) \sigma_{2 s+1}\left(\frac{n-m}{2}\right)\right) \\
& =2 \sum_{s=0}^{k-1}\left(\begin{array}{c}
2 k \\
2 s+1
\end{array}\right) \sum_{m=1}^{n-1} \sigma_{2 k-2 s-1}^{*}(m) \sigma_{2 s+1}^{*}(n-m) \\
& -\left\{\left(\frac{2 k+3}{4 k+2}\right) \sigma_{2 k+1}(n)+\left(\frac{k}{6}-n\right) \sigma_{2 k-1}(n)+\frac{1}{2 k+1} \sum_{j=2}^{k}\left(\begin{array}{c}
2 k+1 \\
2 j
\end{array}\right) B_{2 j} \sigma_{2 k+1-2 j}(n)\right\} \\
& +2\left\{\left(\frac{2 k+3}{4 k+2}\right) \sigma_{2 k+1}\left(\frac{n}{2}\right)+\left(\frac{k}{6}-\frac{n}{2}\right) \sigma_{2 k-1}\left(\frac{n}{2}\right)\right. \\
& \left.+\frac{1}{2 k+1} \sum_{j=2}^{k}\left(\begin{array}{c}
2 k+1 \\
2 j
\end{array}\right) B_{2 j} \sigma_{2 k+1-2 j}\left(\frac{n}{2}\right)\right\} \\
& =\left(\sigma_{2 k+1}^{*}(n)-n \sigma_{2 k-1}^{*}(n)\right)-\left(\frac{2 k+3}{4 k+2}\right)\left\{\sigma_{2 k+1}(n)-2 \sigma_{2 k+1}\left(\frac{n}{2}\right)\right\} \\
& -\frac{k}{6}\left\{\sigma_{2 k-1}(n)-2 \sigma_{2 k-1}\left(\frac{n}{2}\right)\right\}+n\left\{\sigma_{2 k-1}(n)-\sigma_{2 k-1}\left(\frac{n}{2}\right)\right\} \\
& -\frac{1}{2 k+1} \sum_{j=2}^{k}\left(\begin{array}{c}
2 k+1 \\
2 j
\end{array}\right) B_{2 j}\left\{\sigma_{2 k+1-2 j}(n)-2 \sigma_{2 k+1-2 j}\left(\frac{n}{2}\right)\right\} \\
& =\sigma_{2 k+1}^{*}(n)-\left(\frac{2 k+3}{4 k+2}\right) \hat{\sigma}_{2 k+1}(n)-\frac{k}{6} \hat{\sigma}_{2 k-1}(n)-\frac{1}{2 k+1} \sum_{j=2}^{k}\left(\begin{array}{c}
2 k+1 \\
2 j
\end{array}\right) B_{2 j} \hat{\sigma}_{2 k+1-2 j}(n) .
\end{aligned}
$$

This proves the theorem.

Example 7 Let $n$ be a positive integer. In Theorem 1 , put $k=2$, we get

$$
\sum_{m=1}^{n-1} \hat{\sigma}(m) \hat{\sigma}_{3}(n-m)=\frac{1}{8} \sigma_{5}^{*}(n)-\frac{7}{80} \hat{\sigma}_{5}(n)-\frac{1}{24} \hat{\sigma}_{3}(n)+\frac{1}{240} \hat{\sigma}(n) .
$$

Corollary 8 Let $k, n$ be positive integers. Then, we obtain

(i)

$$
\begin{aligned}
\sum_{j=2}^{k}\left(\begin{array}{c}
2 k+1 \\
2 j
\end{array}\right) B_{2 j} \hat{\sigma}_{2 k+1-2 j}(2 n) \\
=\left(\frac{2 k+1}{2}\right) \sigma_{2 k+1}^{*}(2 n)-\left(\frac{2 k+3}{2}\right) \hat{\sigma}_{2 k+1}(2 n)-\left(\frac{k(2 k+1)}{6}\right) \hat{\sigma}_{2 k-1}(2 n) \\
\quad-(2 k+1) \sum_{s=0}^{k-1}\left(\begin{array}{c}
2 k \\
2 s+1
\end{array}\right) \sum_{m=1}^{n-1} \hat{\sigma}_{2 k-2 s-1}(2 m) \hat{\sigma}_{2 s+1}(2 n-2 m)
\end{aligned}
$$


(ii)

$$
\begin{aligned}
\sum_{j=2}^{k}\left(\begin{array}{c}
2 k+1 \\
2 j
\end{array}\right) B_{2 j}\left\{\sigma_{2 k+1-2 j}(n)+\sigma_{2 k+1-2 j}\left(\frac{n}{2}\right)\right\} \\
=-\sigma_{2 k+1}(n)-2(k+1) \sigma_{2 k+1}\left(\frac{n}{2}\right)-\frac{(2 k+1)(k-3 n)}{6} \sigma_{2 k-1}(n) \\
\quad-\frac{(2 k+1)(k-6 n)}{6} \sigma_{2 k-1}\left(\frac{n}{2}\right) \\
+2(2 k+1) \sum_{s=0}^{k-1}\left(\begin{array}{c}
2 k \\
2 s+1
\end{array}\right) \sum_{m=1}^{n-1} \sigma_{2 k-2 s-1}\left(\frac{m}{2}\right) \sigma_{2 s+1}(n-m),
\end{aligned}
$$

(iii)

$$
\begin{aligned}
\sum_{j=2}^{k}\left(\begin{array}{c}
2 k+1 \\
2 j
\end{array}\right) B_{2 j} \sigma_{2 k+1-2 j}^{*}(n) & \\
= & 2 k \sigma_{2 k+1}^{*}(n)-\frac{k(2 k+1)}{6} \sigma_{2 k-1}^{*}(n)-\frac{n(2 k+1)}{2} \hat{\sigma}_{2 k-1}(n) \\
& -2(2 k+1) \sum_{s=0}^{k-1}\left(\begin{array}{c}
2 k \\
2 s+1
\end{array}\right) \sum_{m=1}^{n-1} \sigma_{2 k-2 s-1}^{*}(m) \hat{\sigma}_{2 s+1}(n-m) \\
= & -2(k+1) \sigma_{2 k+1}^{*}(n)-\frac{(k-9 n)(2 k+1)}{6} \sigma_{2 k-1}^{*}(n)+\frac{n(2 k+1)}{2} \sigma_{2 k-1}\left(\frac{n}{2}\right) \\
& +2(2 k+1) \sum_{s=0}^{k-1}\left(\begin{array}{c}
2 k \\
2 s+1
\end{array}\right) \sum_{m=1}^{n-1} \sigma_{2 k-2 s-1}^{*}(m) \sigma_{2 s+1}(n-m) .
\end{aligned}
$$

Proof (i) We note that

$$
\begin{aligned}
\sum_{s=0}^{k-1}\left(\begin{array}{c}
2 k \\
2 s+1
\end{array}\right) \sum_{m=1}^{n-1} \sigma_{2 k-2 s-1}^{*}(2 m) \sigma_{2 s+1}^{*}(n-2 m) \\
=\sum_{s=0}^{k-1}\left(\begin{array}{c}
2 k \\
2 s+1
\end{array}\right) \sum_{m=1}^{n-1} \sigma_{2 k-2 s-1}^{*}(m) \sigma_{2 s+1}^{*}(n-m) \\
\quad-\sum_{s=0}^{k-1}\left(\begin{array}{c}
2 k \\
2 s+1
\end{array}\right) \sum_{m=1}^{n-1} \sigma_{2 k-2 s-1}^{*}(2 m-1) \sigma_{2 s+1}^{*}(n-(2 m-1)) .
\end{aligned}
$$

(ii) and (iii) are applied in a similar way.

\section{Bernoulli polynomials and convolution sums}

Proposition 9 ([12]) Let $k, n$ be positive integers. Then

$$
\begin{aligned}
& \sum_{s=0}^{k-1}\left(\begin{array}{c}
2 k \\
2 s+1
\end{array}\right) \sum_{m=1}^{n-1} 2^{2 k-2 s-1} \sigma_{2 k-2 s-1}(m / 4) \sigma_{2 s+1}(n-m) \\
& \quad=\frac{1}{4} \sigma_{2 k+1}(n / 2)-\frac{1}{4}\left(\sigma_{2 k}(n)-2^{2 k+1} \sigma_{2 k}(n / 2)-2^{2 k} \sigma_{2 k}(n / 4)\right)
\end{aligned}
$$




$$
\begin{aligned}
& -\frac{n}{4}\left(\sigma_{2 k-1}(n)+2^{2 k} \sigma_{2 k-1}(n / 4)\right) \\
& +\frac{1}{2} \sigma_{2 k, 1}(n ; 2)+\frac{2^{2 k-1}}{2 k+1} \sum_{j=0}^{2 k}\left(\begin{array}{c}
2 k+1 \\
j
\end{array}\right) B_{j} \sigma_{2 k+1-j}(n / 2) \\
& +\frac{1}{2(2 k+1)} \sum_{j=0}^{2 k}\left(\begin{array}{c}
2 k+1 \\
j
\end{array}\right) B_{j} 2^{2 k+1-j} \sigma_{2 k+1-j}(n / 4) \\
& +\frac{1}{2(2 k+1)} \sum_{j=0}^{2 k}\left(\begin{array}{c}
2 k+1 \\
j
\end{array}\right) B_{j} \sigma_{2 k+1-j, 1}(n ; 2) \\
& -\frac{1}{2(2 k+1)} \sum_{u+v+w=2 k+1} 2^{v-1}\left(\begin{array}{c}
2 k+1 \\
u, v, w
\end{array}\right) B_{v} \sigma_{w, 1}(n ; 2) .
\end{aligned}
$$

It is well known that $\sigma_{2 k-2 s-1,0}\left(\frac{m}{2} ; 2\right)=2^{2 k-2 s-1} \sigma_{2 k-2 s-1}\left(\frac{m}{4}\right)$. Using Proposition 9, we get this lemma.

Lemma 10 Let $k, n$ be positive integers. Then

(i)

$$
\begin{aligned}
\sum_{s=0}^{k-1}\left(\begin{array}{c}
2 k \\
2 s+1
\end{array}\right) \sum_{m=1}^{n-1} \sigma_{2 k-2 s-1,0}\left(\frac{m}{2} ; 2\right) \sigma_{2 s+1}(n-m) \\
=\frac{1}{4} \sigma_{2 k+1}\left(\frac{n}{2}\right)+\frac{1}{4(2 k+1)} \sigma_{2 k+1,0}(n ; 2)+\frac{1}{2(2 k+1)} \sigma_{2 k+1,0}\left(\frac{n}{2} ; 2\right) \\
\quad+\frac{1}{2(2 k+1)} \sigma_{2 k, 1}(n ; 2)-\frac{1}{4} \sigma_{2 k}(n)+\frac{1}{2} \sigma_{2 k, 0}(n ; 2)-\frac{1}{4} \sigma_{2 k, 0}(n ; 2)+\frac{1}{4} \sigma_{2 k, 1}(n ; 2) \\
\quad-\frac{n}{4} \sigma_{2 k-1}(n)+\frac{k}{6} \sigma_{2 k-1,0}(n ; 2)+\left(\frac{k}{12}-\frac{n}{8}\right) \sigma_{2 k-1,0}\left(\frac{n}{2} ; 2\right) \\
+\frac{k}{12} \sigma_{2 k-1,1}(n ; 2)+\frac{2^{2 k}}{2(2 k+1)} \sum_{j=2}^{k}\left(\begin{array}{c}
2 k+1 \\
2 j
\end{array}\right) B_{2 j} \sigma_{2 k+1-2 j}\left(\frac{n}{2}\right) \\
\quad+\frac{1}{2(2 k+1)} \sum_{j=2}^{k}\left(\begin{array}{c}
2 k+1 \\
2 j
\end{array}\right) B_{2 j} \sigma_{2 k+1-2 j, 0}\left(\frac{n}{2} ; 2\right) \\
\quad+\frac{1}{2(2 k+1)} \sum_{j=2}^{k}\left(\begin{array}{c}
2 k+1 \\
2 j
\end{array}\right) B_{2 j} \sigma_{2 k+1-2 j, 1}(n ; 2) \\
\quad-\frac{1}{2(2 k+1)} \sum_{u+v+w=2 k+1} 2^{v-1}\left(\begin{array}{c}
2 k+1 \\
u, v, w
\end{array}\right) B_{v} \sigma_{w, 1}(n ; 2),
\end{aligned}
$$

(ii)

$$
\begin{aligned}
\sum_{s=0}^{k-1}\left(\begin{array}{c}
2 k \\
2 s+1
\end{array}\right) \sum_{m=1}^{n-1} \sigma_{2 k-2 s-1,1}\left(\frac{m}{2} ; 2\right) \sigma_{2 s+1}(n-m) \\
=\left(\frac{1}{2(2 k+1)}\right) \sigma_{2 k+1}(n)+\left(\frac{k+1}{2 k+1}\right) \sigma_{2 k+1}\left(\frac{n}{2}\right)+\left(\frac{k-3 n}{12}\right) \sigma_{2 k-1}(n) \\
\quad+\left(\frac{k-6 n}{12}\right) \sigma_{2 k-1}\left(\frac{n}{2}\right)-\frac{1}{4} \sigma_{2 k+1}\left(\frac{n}{2}\right)
\end{aligned}
$$




$$
\begin{aligned}
& -\left(\frac{1}{4(2 k+1)}\right) \sigma_{2 k+1,0}(n ; 2)-\left(\frac{1}{2(2 k+1)}\right) \sigma_{2 k+1,0}\left(\frac{n}{2} ; 2\right) \\
& -\left(\frac{1}{2(2 k+1)}\right) \sigma_{2 k, 1}(n ; 2)+\frac{1}{4} \sigma_{2 k}(n) \\
& -\frac{1}{2} \sigma_{2 k, 0}(n ; 2)+\frac{1}{4} \sigma_{2 k, 0}(n ; 2)-\frac{1}{4} \sigma_{2 k, 1}(n ; 2) \\
& +\frac{n}{4} \sigma_{2 k-1}(n)-\frac{k}{6} \sigma_{2 k-1,0}(n ; 2)-\left(\frac{k}{12}-\frac{n}{8}\right) \sigma_{2 k-1,0}\left(\frac{n}{2} ; 2\right)-\frac{k}{12} \sigma_{2 k-1,1}(n ; 2) \\
& -\frac{2^{2 k}}{2(2 k+1)} \sum_{j=2}^{k}\left(\begin{array}{c}
2 k+1 \\
2 j
\end{array}\right) B_{2 j} \sigma_{2 k+1-2 j}\left(\frac{n}{2}\right) \\
& -\frac{1}{2(2 k+1)} \sum_{j=2}^{k}\left(\begin{array}{c}
2 k+1 \\
2 j
\end{array}\right) B_{2 j} \sigma_{2 k+1-2 j, 0}\left(\frac{n}{2} ; 2\right) \\
& -\frac{1}{2(2 k+1)} \sum_{j=2}^{k}\left(\begin{array}{c}
2 k+1 \\
2 j
\end{array}\right) B_{2 j} \sigma_{2 k+1-2 j, 1}(n ; 2) \\
& +\frac{1}{2(2 k+1)} \sum_{u+v+w=2 k+1} 2^{v-1}\left(\begin{array}{c}
2 k+1 \\
u, v, w
\end{array}\right) B_{v} \sigma_{w, 1}(n ; 2) .
\end{aligned}
$$

Remark 11 (i) Using Lemma 10, we obtain

$$
\begin{aligned}
& \sum_{u+v+w=2 k+1} 2^{v-1}\left(\begin{array}{l}
2 k+1 \\
u, v, w
\end{array}\right) B_{v} \sigma_{w, 1}(n ; 2) \\
& =\left(\frac{2 k+1}{2}\right)\left\{\sigma_{2 k+1}\left(\frac{n}{2}\right)-\sigma_{2 k}(n)+2 \sigma_{2 k, 0}(n ; 2)+\sigma_{2 k, 0}\left(\frac{n}{2} ; 2\right)\right\} \\
& -\left(\frac{n(2 k+1)}{2}\right)\left\{\sigma_{2 k-1}(n)+2 \sigma_{2 k-1,0}\left(\frac{n}{2} ; 2\right)\right\}+(2 k+1) \sigma_{2 k, 1}(n ; 2) \\
& +2^{2 k} \sum_{j=0}^{2 k}\left(\begin{array}{c}
2 k+1 \\
j
\end{array}\right) B_{j} \sigma_{2 k+1-j}\left(\frac{n}{2}\right)+\sum_{j=0}^{2 k}\left(\begin{array}{c}
2 k+1 \\
j
\end{array}\right) B_{j} \sigma_{2 k+1-j, 0}\left(\frac{n}{2} ; 2\right) \\
& +\sum_{j=0}^{2 k}\left(\begin{array}{c}
2 k+1 \\
j
\end{array}\right) B_{j} \sigma_{2 k+1-j, 1}(n ; 2) \\
& -2(2 k+1) \sum_{s=0}^{k-1}\left(\begin{array}{c}
2 k \\
2 s+1
\end{array}\right) \sum_{m=1}^{n-1} \sigma_{2 k-2 s-1,0}\left(\frac{m}{2} ; 2\right) \sigma_{2 s+1}(n-m) \\
& =-\left(\frac{2 k+1}{2}\right)\left\{\sigma_{2 k+1}\left(\frac{n}{2}\right)-2 n \sigma_{2 k-1,1}\left(\frac{n}{2} ; 2\right)+\sigma_{2 k, 1}\left(\frac{n}{2} ; 2\right)\right\} \\
& -\sum_{j=0}^{2 k}\left(\begin{array}{c}
2 k+1 \\
j
\end{array}\right) B_{j} \sigma_{2 k+1-j}(n)+\left(2^{2 k}-1\right) \sum_{j=0}^{2 k}\left(\begin{array}{c}
2 k+1 \\
j
\end{array}\right) B_{j} \sigma_{2 k+1-j}\left(\frac{n}{2}\right) \\
& +\sum_{j=0}^{2 k}\left(\begin{array}{c}
2 k+1 \\
j
\end{array}\right) B_{j} \sigma_{2 k+1-j, 0}\left(\frac{n}{2} ; 2\right)+\sum_{j=0}^{2 k}\left(\begin{array}{c}
2 k+1 \\
j
\end{array}\right) B_{j} \sigma_{2 k+1-j, 1}(n ; 2) \\
& +2(2 k+1) \sum_{s=0}^{k-1}\left(\begin{array}{c}
2 k \\
2 s+1
\end{array}\right) \sum_{m=1}^{n-1} \sigma_{2 k-2 s-1,1}\left(\frac{m}{2} ; 2\right) \sigma_{2 s+1}(n-m) .
\end{aligned}
$$


(ii) If $n$ is an odd integer, then

$$
\begin{aligned}
& \sum_{u+v+w=2 k+1} 2^{v-1}\left(\begin{array}{l}
2 k+1 \\
u, v, w
\end{array}\right) B_{v} \sigma_{w, 1}(n ; 2) \\
& =2(2 k+1) \sum_{s=0}^{k-1}\left(\begin{array}{c}
2 k \\
2 s+1
\end{array}\right) \sum_{m=1}^{n-1} \sigma_{2 k-2 s-1,1}\left(\frac{m}{2} ; 2\right) \sigma_{2 s+1}(n-m) .
\end{aligned}
$$

(iii) In (3.1), put $k=1$, we get

$$
\sum_{u+v+w=3} 2^{\nu-1}\left(\begin{array}{c}
3 \\
u, v, w
\end{array}\right) B_{v} \sigma_{w, 1}(n ; 2)=12 \sum_{m=1}^{n-1} \sigma_{1,1}\left(\frac{m}{2} ; 2\right) \sigma_{1}(n-m),
$$

and thus,

$$
\sum_{m=1}^{n-1} \sigma_{1,1}\left(\frac{m}{2} ; 2\right) \sigma_{1}(n-m)=\frac{1}{24}\left(\sigma_{3}(n)-\sigma_{1}(n)\right) .
$$

In (3.1), replace $k$ by 2 , we find that

$$
\begin{aligned}
& \sum_{u+v+w=5} 2^{v-1}\left(\begin{array}{c}
5 \\
u, v, w
\end{array}\right) B_{v} \sigma_{w, 1}(n ; 2) \\
& =40\left(\sum_{m=1}^{n-1} \sigma_{3,1}\left(\frac{m}{2} ; 2\right) \sigma_{1}(n-m)+\sum_{m=1}^{n-1} \sigma_{1,1}\left(\frac{m}{2} ; 2\right) \sigma_{3}(n-m)\right),
\end{aligned}
$$

and thus,

$$
\begin{aligned}
& \sum_{m=1}^{n-1} \sigma_{3,1}\left(\frac{m}{2} ; 2\right) \sigma_{1}(n-m)+\sum_{m=1}^{n-1} \sigma_{1,1}\left(\frac{m}{2} ; 2\right) \sigma_{3}(n-m) \\
& \quad=\frac{1}{240}\left(3 \sigma_{5}(n)-10 \sigma_{3}(n)+7 \sigma_{1}(n)\right) .
\end{aligned}
$$

Proof of Theorem 3 If $n=1$, compare both sides of (3.1), we obtain

$$
\sum_{u+v+w=2 k+1} 2^{v-1}\left(\begin{array}{l}
2 k+1 \\
u, v, w
\end{array}\right) B_{v}=0 .
$$

If we put $n=3$ in (3.1), we obtain

$$
\begin{aligned}
& \sum_{u+v+w=2 k+1} 2^{v-1}\left(\begin{array}{l}
2 k+1 \\
u, v, w
\end{array}\right) B_{v} \cdot\left(1+3^{w}\right) \\
& =\sum_{u+v+w=2 k+1} 2^{v-1}\left(\begin{array}{c}
2 k+1 \\
u, v, w
\end{array}\right) B_{v} \cdot 3^{w} \\
& =2(2 k+1) \sum_{s=0}^{k-1}\left(\begin{array}{c}
2 k \\
2 s+1
\end{array}\right) .
\end{aligned}
$$


From (3.1) and (3.3), we get

$$
\sum_{u+v+w=2 k+1} 2^{v-1}\left(\begin{array}{l}
2 k+1 \\
u, v, w
\end{array}\right) B_{v} \cdot 3^{w}=(2 k+1) 2^{2 k}
$$

By combining (1.1) and (3.4), we obtain

$$
\sum_{u+v+w=2 k+1} 2^{v-1}\left(\begin{array}{c}
2 k+1 \\
u, v, w
\end{array}\right) B_{v} \cdot 3^{w}=2^{2 k} B_{2 k+1}(2) .
$$

Others cases follow in a similar way. This completes the proof.

Proof of Theorem 4 (i) If $a$ is a positive integer, then

$$
\sum_{u+v+w=2 k+1} 2^{v-1}\left(\begin{array}{c}
2 k+1 \\
u, v, w
\end{array}\right) B_{v} \cdot \sigma_{w, 1}\left(2^{a} ; 2\right)=\sum_{u+v+w=2 k+1} 2^{v-1}\left(\begin{array}{l}
2 k+1 \\
u, v, w
\end{array}\right) B_{v}=0
$$

by (3.2). According to Remark 11(i), we deduce that

$$
\begin{aligned}
0= & 2(2 k+1) \sum_{s=0}^{k-1}\left(\begin{array}{c}
2 k \\
2 s+1
\end{array}\right) \sum_{m=1}^{2^{a}-1} \sigma_{2 k-2 s-1,1}\left(\frac{m}{2} ; 2\right) \sigma_{2 s+1}\left(2^{a}-m\right) \\
& -\sum_{j=0}^{2 k}\left(\begin{array}{c}
2 k+1 \\
j
\end{array}\right) B_{j} \sigma_{2 k+1-j}\left(2^{a}\right)+\left(2^{2 k}-1\right) \sum_{j=0}^{2 k}\left(\begin{array}{c}
2 k+1 \\
j
\end{array}\right) B_{j} \sigma_{2 k+1-j}\left(\frac{2^{a}}{2}\right) \\
& +\sum_{j=0}^{2 k}\left(\begin{array}{c}
2 k+1 \\
j
\end{array}\right) B_{j} \sigma_{2 k+1-j, 0}\left(\frac{2^{a}}{2} ; 2\right)-\left(\frac{2 k+1}{2}\right)\left\{\sigma_{2 k+1}\left(\frac{2^{a}}{2}\right)-2\left(2^{a}\right)+1\right\} .
\end{aligned}
$$

If $a=1$, it is clearly evident. We suppose that $a>1$. We check that

$$
\begin{aligned}
& \sum_{j=0}^{2 k}\left(\begin{array}{c}
2 k+1 \\
j
\end{array}\right) B_{j} \cdot\left(2^{a}\right)^{2 k+1-j} \\
& \quad=\sum_{j=0}^{2 k}\left(\begin{array}{c}
2 k+1 \\
j
\end{array}\right)(-1)^{j} B_{j} \cdot\left(2^{a}\right)^{2 k+1-j}+2\left(\begin{array}{c}
2 k+1 \\
1
\end{array}\right) B_{1} \cdot\left(2^{a}\right)^{2 k} \\
& =(2 k+1) \sum_{j=0}^{2^{a}} j^{2 k}+2(2 k+1)\left(\frac{-1}{2}\right)\left(2^{a}\right)^{2 k} \\
& =B_{2 k+1}\left(2^{a}\right)
\end{aligned}
$$

by (1.1).

(ii) and (iii) are applied in a similar way.

Remark 12 If $p$ is a prime integer, then

$$
\sum_{u+v+w=2 k+1} 2^{v-2}\left(\begin{array}{c}
2 k+1 \\
u, v, w
\end{array}\right) B_{v} \cdot p^{w}=(2 k+1) \sum_{s=0}^{k-1}\left(\begin{array}{c}
2 k \\
2 s+1
\end{array}\right) \sum_{m=1}^{p-1} \sigma_{2 k-2 s-1,1}\left(\frac{m}{2} ; 2\right) \sigma_{2 s+1}(p-m)
$$

by (3.1) and (3.2). 


\section{Competing interests}

The authors declare that they have no competing interests.

\section{Authors' contributions}

All authors contributed equally and significantly in writing this article. All authors read and approved the final manuscript.

\section{Author details}

${ }^{1}$ National Institute for Mathematical Sciences, Yuseong-daero 1689-gil, Yuseong-gu, Daejeon, 305-811, South Korea.

${ }^{2}$ Department of Elementary Mathematics Education, Necatibey Faculty of Education, Balikesir University, Balikesir, 10100, Turkey.

Received: 26 July 2013 Accepted: 23 September 2013 Published: 08 Nov 2013

\section{References}

1. Hahn, H: Convolution sums of some functions on divisors. Rocky Mt. J. Math. 37(5), 1593-1622 (2007)

2. Besge, M: Extrait d'une lettre de M. Besge à M. Liouville. J. Math. Pures Appl. 7, 375-376 (1862)

3. Cheng, N, Williams, KS: Evaluation of some convolution sums involving the sum of divisors functions. Yokohama Math. J. 52, 39-57 (2005)

4. Lahiri, DB: On Ramanujan's function $\tau(n)$ and the divisor function $\sigma(n)$, I. Bull. Calcutta Math. Soc. 38, 193-206 (1946)

5. Melfi, G: On some modular identities. In: Number Theory (Eger, 1996), pp. 371-382. de Gruyter, Berlin (1998)

6. Huard, JG, Ou, ZM, Spearman, BK, Williams, KS: Elementary evaluation of certain convolution sums involving divisor functions. In: Number Theory for the Millennium, vol. II, pp. 229-274 (2002)

7. Kim, D, Kim, A, Sankaranarayanan, A: Bernoulli numbers, convolution sums and congruences of coefficients for certain generating functions. J. Inequal. Appl. 2013, 225 (2013)

8. Williams, KS: Number Theory in the Spirit of Liouville. London Mathematical Society Student Texts, vol. 76. Cambridge University Press, Cambridge (2011)

9. Kim, D, Kim, A, Kim, M: A remark on algebraic curves derived from convolution sums. J. Inequal. Appl. 2013,58 (2013)

10. Simsek, Y: Elliptic analogue of the Hardy sums related to elliptic Bernoulli functions. Gen. Math. 15(2-3), 3-23 (2007)

11. Kim, D, Bayad, A: Convolution identities for twisted Eisenstein series and twisted divisor functions. Fixed Point Theory Appl. 2013, 81 (2013)

12. Cho, B, Kim, D, Park, H: Combinatorial convolution sums derived from divisor functions and Faulhaber sums (2013, submitted)

10.1186/1687-1847-2013-310

Cite this article as: Kim and Yildiz Ikikardes: Certain combinatoric Bernoulli polynomials and convolution sums of divisor functions. Advances in Difference Equations 2013, 2013:310

\section{Submit your manuscript to a SpringerOpen ${ }^{\circ}$ journal and benefit from:}

- Convenient online submission

Rigorous peer review

- Immediate publication on acceptance

- Open access: articles freely available online

- High visibility within the field

- Retaining the copyright to your article 